\title{
Seletividade fisiológica de inseticidas em duas espécies de crisopídeos
}

\author{
Maurício Sekiguchi Godoy(1), Geraldo Andrade Carvalho(1), Beatriz Ferreira Carvalho(1) e Olinto Lasmar ${ }^{(1)}$ \\ (1)Universidade Federal de Lavras, Departamento de Entomologia, Caixa Postal 3037, CEP 37200-000 Lavras, MG. \\ E-mail: msdgodoy@yahoo.com.br, gacarval@den.ufla.br, beatrizfcarvalho@uol.com.br, lasmar84@yahoo.com.br
}

\begin{abstract}
Resumo - O objetivo deste trabalho foi avaliar a seletividade de inseticidas recentemente lançados no mercado para o controle de pragas na cultura dos citros, em adultos de Chrysoperla externa e Ceraeochrysa cubana.

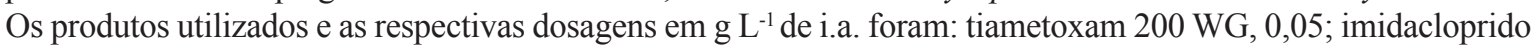
$700 \mathrm{WG}, 0,07$; milbemectina $50 \mathrm{CE}, 0,008$; piriproxifem $100 \mathrm{CE}, 0,075$; e espirodiclofeno $240 \mathrm{SC}, 0,06$. Em cada tratamento, 11 casais de $C$. externa e 13 de C. cubana, com idade de até 24 horas, foram submetidos à aplicação dos inseticidas e de água (testemunha), por meio de torre de Potter. A mortalidade dos adultos, a capacidade de oviposição num período de oito semanas e a viabilidade dos ovos foram avaliadas. O tiametoxam foi classificado como nocivo a C. externa; o imidacloprido como moderadamente nocivo e os demais inseticidas como inócuos. Para C. cubana, o tiametoxam foi considerado nocivo, o espirodiclofeno foi levemente nocivo e os demais produtos foram inócuos. Os produtos milbemectina, piriproxifem e espirodiclofeno podem ser recomendados em programas de manejo integrado de pragas visando à manutenção dessas espécies de artrópodes benéficos em áreas de citricultura.
\end{abstract}

Termos para indexação: Ceraeochrysa cubana, Citrus, Chrysoperla externa, artrópodes benéficos, manejo integrado de pragas, toxicidade.

\section{Physiological selectivity of insecticides to two lacewing species}

\begin{abstract}
The objective of this work was to evaluate the effects of insecticides recently released for the control of citrus pests on adult specimens of Chrysoperla externa and Ceraeochrysa cubana. The products used and their respective dosages in $\mathrm{g} \mathrm{L}^{-1}$ of a.i. were: thiametoxam $200 \mathrm{WG}, 0.05$; imidacloprid $700 \mathrm{WG}, 0.07$; milbemectin $50 \mathrm{CE}, 0.008$; pyriproxyfen $100 \mathrm{CE}, 0.075$; and spirodiclofen $240 \mathrm{SC}, 0.06$. In each treatment, 11 couples of C. externa and 13 of C. cubana, at an age up to 24 hours, were submitted to the application of the insecticides and water (control), through a Potter's tower. Adults mortality and oviposition capacity were evaluated in a period of eight weeks, as well as the viability of laid eggs. Thiametoxam was classified as harmful to C. externa; imidacloprid as moderately harmful and the other insecticides as harmless. As for C. cubana, thiametoxam was considered harmful; spirodiclofen was slightly harmful and the other products were innocuous. The insecticides milbemectin, pyriproxyfen and spirodiclofen can be recommended to integrated pest management programs aiming at the maintenance of these species of beneficial arthropods in citrus areas.
\end{abstract}

Index terms: Ceraeochrysa cubana, Citrus, Chrysoperla externa, beneficial arthropods, integrated pest management, toxicity.

\section{Introdução}

Os insetos-praga que acometem a cultura dos citros causam lesões às folhas, frutos e ramos, além de serem disseminadores de doenças (Ribeiro et al., 2007). Atualmente, com a produção integrada de frutas (PIF), prioriza-se a utilização de táticas de controle de pragas que preservem e incrementem fatores de mortalidade natural por meio da utilização de agentes de controle biológico (Moura et al., 2009).
O uso indiscriminado de inseticidas pode causar contaminação ambiental, bem como prejudicar artrópodes benéficos, como os inimigos naturais e polinizadores. Por isso, o conhecimento da seletividade de inseticidas é fundamental para implementação de programas de manejo integrado de pragas (MIP) (Carvalho et al., 2007). Produtos seletivos são prejudiciais às pragas e pouco tóxicos aos organismos benéficos (Ripper et al., 1951). Entre os inimigos naturais, artrópodes da ordem 
Neuroptera, principalmente da família Chrysopidae, são considerados predadores de pragas de muitas culturas economicamente rentáveis, como citros. Eles podem se alimentar de ovos, lagartas neonatas, pulgões, cochonilhas, ácaros e vários outros artrópodes de pequeno tamanho e de tegumento facilmente perfurável (Carvalho \& Souza, 2000).

Entre as espécies de crisopídeos, Chrysoperla externa (Hagen, 1861) (Neuroptera: Chrysopidae) destacase como uma das mais frequentes em pomares de citros, e pode ser encontrada nas copas das árvores e, principalmente, na vegetação entre as ruas (Bezerra et al., 2006). De acordo com Souza et al. (1996) e Gitirana Neto et al. (2001), outra espécie de crisopídeo comum em cultura de citros é a Ceraeochrysa cubana (Hagen, 1861) (Neuroptera: Chrysopidae), cujas larvas predam pulgões, cochonilhas, mosca-branca e ácaros.

No que se refere aos efeitos de pesticidas sobre espécies de predadores, Schuster \& Stansly (2000) observaram que larvas de $C$. cubana foram tolerantes a resíduos do piretroide bifentrina. Godoy et al. (2004) constataram que tiacloprido, deltametrina, lufenurom, tebufenozide, óxido de fenbutatina e abamectina, nas maiores doses recomendas pelos fabricantes, foram seletivos a pupas de C. externa, quando submetidas à pulverização direta. Dessa forma, entre os vários compostos aplicados na cultura de citros, é possível que alguns possam mostrar-se inócuos a certas espécies de crisopídeos em razão da seletividade fisiológica, na qual geralmente estão envolvidos processos de absorção, penetração, transporte e ativação dos compostos, que resultam em toxicidade diferencial de inseticidas a diferentes organismos-alvo. Há escassez de pesquisas a respeito dos efeitos desses produtos sobre essas espécies de crisopídeos em condições brasileiras.

O objetivo deste trabalho foi verificar a seletividade de inseticidas recentemente lançados no mercado para o controle de pragas na cultura dos citros em adultos de Chrysoperla externa e Ceraeochrysa cubana.

\section{Material e Métodos}

Os bioensaios foram conduzidos no Laboratório de Estudos de Seletividade do Departamento de Entomologia da Universidade Federal de Lavras, durante o ano de 2009. A criação de C. externa e C. cubana foi realizada empregando-se as metodologias utilizadas por Ribeiro et al. (1991) e Costa et al.
(2003). Os insetos foram mantidos em sala climatizada a $25 \pm 2^{\circ} \mathrm{C}$, UR de $70 \pm 10 \%$ e fotófase de 14 horas.

Os bioensaios foram realizados de acordo com as recomendações metodológicas preconizadas pela Organização Internacional para o Controle Biológico e Integrado de Plantas e Animais Nocivos (IOBC) (International Organization for Biological Control, 1992). Os produtos avaliados com suas respectivas doses em $\mathrm{g} \mathrm{L}^{-1}$ de i.a. foram: tiametoxam $200 \mathrm{WG}, 0,05$; imidacloprido $700 \mathrm{WG}, 0,07$; milbemectina $50 \mathrm{CE}$, 0,008; piriproxifem $100 \mathrm{CE}, 0,075$; e espirodiclofeno 240 SC, 0,06 . As concentrações utilizadas correspondem às mais elevadas recomendadas pelos fabricantes para o controle de pragas na cultura de citros. Água destilada foi utilizada no tratamento testemunha.

Onze e treze casais de adultos da geração $F_{3}$ de C. externa e C. cubana, respectivamente, com idade de até 24 horas e obtidos da criação em laboratório, foram anestesiados com $\mathrm{CO}_{2}$ e imediatamente submetidos à pulverização dos compostos em torre de Potter regulada à pressão de $15 \mathrm{lb} \mathrm{pol}^{-2}$, com um volume médio de aplicação de $1,5 \pm 0,5 \mathrm{mg} \mathrm{cm}^{-2}$. Após a pulverização, cada casal foi individualizado em gaiola de cloreto de polivinila (PVC) de $10 \mathrm{~cm}$ de diâmetro por $10 \mathrm{~cm}$ de altura, revestida internamente com papel-filtro. Cada conjunto foi mantido em sala climatizada a $25 \pm 2{ }^{\circ} \mathrm{C}$, UR de $70 \pm 10 \%$ e fotófase de 14 horas. Os adultos receberam, a cada dois dias, água e dieta artificial composta de levedo de cerveja e mel $(1: 1, \mathrm{v} / \mathrm{v})$.

Os bioensaios foram realizados em delineamento inteiramente ao acaso com seis tratamentos, contendo 11 repetições de $C$. externa e 13 de $C$. cubana, cada uma formada por um casal do predador. A mortalidade dos adultos, a capacidade de oviposição, a fertilidade e a viabilidade dos ovos foram avaliadas durante oito semanas, após a aplicação dos inseticidas. Após o período de pré-oviposição, de aproximadamente sete dias, foram realizadas três coletas semanais de ovos, relativas a cada tratamento, de modo a avaliar a viabilidade. Foram coletados 100 ovos, os quais foram individualizados em compartimentos de placas de microtitulação tipo Elisa, fechadas com PVC laminado e mantidas em sala climatizada, conforme descrito, durante seis dias. Após esse período, efetuou-se a avaliação do número de ovos viáveis.

Os dados obtidos foram submetidos a técnicas de análise de sobrevivência, e os tempos de permanência em cada estágio - ovo, larva, pupa e adulto - 
permitiram calcular o último tempo de morte (UTM). Além disso, também foi possível construir a curva de sobrevivência, com o estimador Kaplan-Meier, para a função sobrevivência, tendo-se utilizado o software R versão 2.6.0 (R Development Core Team, 2008). Considerou-se como UTM o evento em que ocorreu a última mortalidade do predador dentro da população em estudo.

Os dados referentes ao número e viabilidade de ovos foram submetidos à análise de variância, e as médias foram comparadas pelo teste de Tukey (1965), a 5\% de probabilidade, com uso do programa SAS (SAS Institute, 2001). A toxicidade dos produtos foi calculada em função do efeito total (E) de cada produto, com base na mortalidade dos insetos até 24 horas após a exposição aos produtos e na redução de sua capacidade reprodutiva, de acordo com a fórmula proposta por Vogt (1992). Os produtos foram enquadrados em classes de toxicidade conforme Hassan \& Degrande (1996), da seguinte maneira: classe 1, inócuo ( $\mathrm{E}<30 \%)$; classe 2 , levemente nocivo $(30 \% \leq \mathrm{E} \leq 79 \%)$; classe 3 , moderadamente nocivo $(80 \% \leq \mathrm{E} \leq 99 \%)$ e classe 4 , nocivo ( $\mathrm{E}>99 \%)$.

\section{Resultados e Discussão}

Houve diferença significativa entre os tratamentos no que se refere à probabilidade de sobrevivência $[\mathrm{P}(\mathrm{S})]$ de C. externa, e o tiametoxam resultou em $\mathrm{P}(\mathrm{S})$ de apenas $22,7 \%$ aos quatro dias, o que sugere alta toxicidade deste produto aos adultos dessa espécie (Tabela 1, Figura 1). Resultados semelhantes foram obtidos por Bueno \& Freitas (2003), os quais verificaram que imidacloprido, ingrediente ativo pertencente ao mesmo grupo químico do tiametoxam (neonicotinoide), causou $100 \%$ de mortalidade de larvas de primeiro instar de C. externa. Rocha (2008), ao estudar a seletividade de imidacloprido $\left(0,7 \mathrm{~g} \mathrm{~L}^{-1}\right.$ de i.a.) e tiametoxam $\left(0,5 \mathrm{~g} \mathrm{~L}^{-1}\right.$ de i.a.) em adultos de $C$. externa coletados em lavoura de café, relatou que esses compostos provocaram $100 \%$ de mortalidade dos insetos e impossibilitaram a realização de avaliações da fertilidade e viabilidade de ovos de gerações subsequentes.

Nos demais tratamentos, os adultos de C. externa apresentaram $40,9 \%$ de $\mathrm{P}(\mathrm{S})$, próximo ou no final dos eventos (58 e 60 dias), o que revela baixa toxicidade dos produtos. Moura et al. (2009) também verificaram baixa toxicidade de produtos a duas populações de adultos de C. externa, que observaram mortalidades de no máximo $6,7 \%$ causadas por enxofre, abamectina e triclorfom.

É importante salientar que o imidacloprido ocasionou mortalidades nos primeiros dias após a aplicação, o que compromete a capacidade de a predação realizada por C. externa ser benéfica à cultura (Figura 1). Isso foi evidenciado após as avaliações do número e viabilidade de ovos, em que esses parâmetros biológicos apresentaram menores médias em comparação ao tratamento testemunha (Tabela 1).

Estudos demonstraram que alguns neonicotinoides podem apresentar maior capacidade de penetração na cutícula dos insetos e se acumular em seus organismos, por exemplo, nos tecidos gordurosos (Tomizawa \& Casida, 2005). Esse fato pode afetar o desenvolvimento dos organismos, bem como comprometer a reprodução de gerações subsequentes.

Com relação aos demais produtos avaliados, a milbemectina também interferiu no número de ovos, e ocasionou a segunda menor média (Tabela 1). Quanto à viabilidade, milbemectina e piriproxifem apresentaram as maiores médias, e imidacloprido e espirodiclofeno

Tabela 1. Último tempo de morte (UTM), probabilidade de sobrevivência $[\mathrm{P}(\mathrm{S})]$ e médias \pm erro-padrão do número de ovos e de sua viabilidade, para adultos de Chrysoperla externa e Ceraeochrysa cubana tratados diretamente com os produtos químicos ${ }^{(1)}$.

\begin{tabular}{lcccc}
\hline Tratamento & $\begin{array}{c}\text { UTM } \\
\text { (dias) }\end{array}$ & $\begin{array}{c}\text { P(S) } \\
(\%)\end{array}$ & № de ovos & $\begin{array}{c}\text { Viabilidade } \\
(\%)\end{array}$ \\
\hline Tiametoxam & 4 & 22,7 & Chrysoperla externa & - \\
Imidacloprido & 60 & 40,9 & $11,00 \pm 2,14 \mathrm{~d}$ & $36,00 \pm 1,07 \mathrm{~d}$ \\
Milbemectina & 60 & 40,9 & $43,04 \pm 2,14 \mathrm{c}$ & $92,25 \pm 1,07 \mathrm{~b}$ \\
Piriproxifem & 58 & 40,9 & $65,73 \pm 2,14 \mathrm{a}$ & $96,08 \pm 1,07 \mathrm{ab}$ \\
Espirodiclofeno & 58 & 40,9 & $56,99 \pm 2,14 \mathrm{~b}$ & $78,75 \pm 1,07 \mathrm{c}$ \\
Testemunha & 60 & 40,9 & $51,59 \pm 2,14 \mathrm{~b}$ & $99,00 \pm 1,07 \mathrm{a}$ \\
\hline CV (\%) & - & - & 38,48 & 14,59 \\
\hline Log-Rank (p-valor) & $<0,0001$ & - & - \\
\hline & \multicolumn{5}{c}{ Ceraeochrysa cubana } \\
Tiametoxam & 1 & 0 & - & - \\
Imidacloprido & 28 & 65,4 & $31,36 \pm 1,83 \mathrm{~b}$ & $98,58 \pm 0,36 \mathrm{ab}$ \\
Milbemectina & 30 & 65,4 & $29,25 \pm 1,83 \mathrm{bc}$ & $96,25 \pm 0,36 \mathrm{c}$ \\
Piriproxifem & 19 & 73,1 & $28,56 \pm 1,83 \mathrm{bc}$ & $98,17 \pm 0,36 \mathrm{~b}$ \\
Espirodiclofeno & 21 & 80,8 & $22,84 \pm 1,83 \mathrm{c}$ & $98,42 \pm 0,36 \mathrm{~b}$ \\
Testemunha & 26 & 84,6 & $37,58 \pm 1,83 \mathrm{a}$ & $99,83 \pm 0,36 \mathrm{a}$ \\
\hline CV (\%) & - & - & 42,48 & 3,99 \\
\hline Log-Rank (p-valor) & $<0,0001$ & - & - \\
\hline
\end{tabular}

${ }^{(1)}$ Médias seguidas de letras iguais nas colunas não diferem entre si pelo teste Tukey, a 5\% de probabilidade. 
proporcionaram as menores percentagens de viabilidade de ovos. Resultados semelhantes foram verificados para adultos de $C$. externa submetidos a abamectina (Godoy et al., 2004; Moura et al., 2009), produto do mesmo grupo químico de milbemectina. Esses autores relataram diminuição na capacidade de oviposição dessa espécie, porém, em relação à viabilidade dos ovos, não foram observadas reduções significativas.

$\mathrm{Na}$ análise de sobrevivência de adultos de C. cubana, observaram-se respostas diferenciadas entre os tratamentos (Tabela 1, Figura 1). O tiametoxam apresentou-se muito tóxico a adultos dessa espécie, com 100\% de mortalidade em apenas um dia após pulverização sobre os insetos. Santa-Cecília et al. (1997), ao avaliar outros grupos de compostos químicos sobre populações de adultos de C. cubana, também obtiveram $100 \%$ de mortalidade dos espécimens em apenas 24 horas após a aplicação de fempropatrina $\left(0,15 \mathrm{~g} \mathrm{~L}^{-1}\right.$ de i.a.) e fenitrotiom $\left(0,75 \mathrm{~g} \mathrm{~L}^{-1}\right.$ de i.a.).

Os demais inseticidas testados em C. cubana possibilitaram estudos até o término das avaliações. $\mathrm{O}$ espirodiclofeno foi o inseticida que resultou nos melhores índices: $\mathrm{P}(\mathrm{S})$ de $80,8 \%$ e UTM no vigésimo primeiro dia. Levando-se em consideração que os
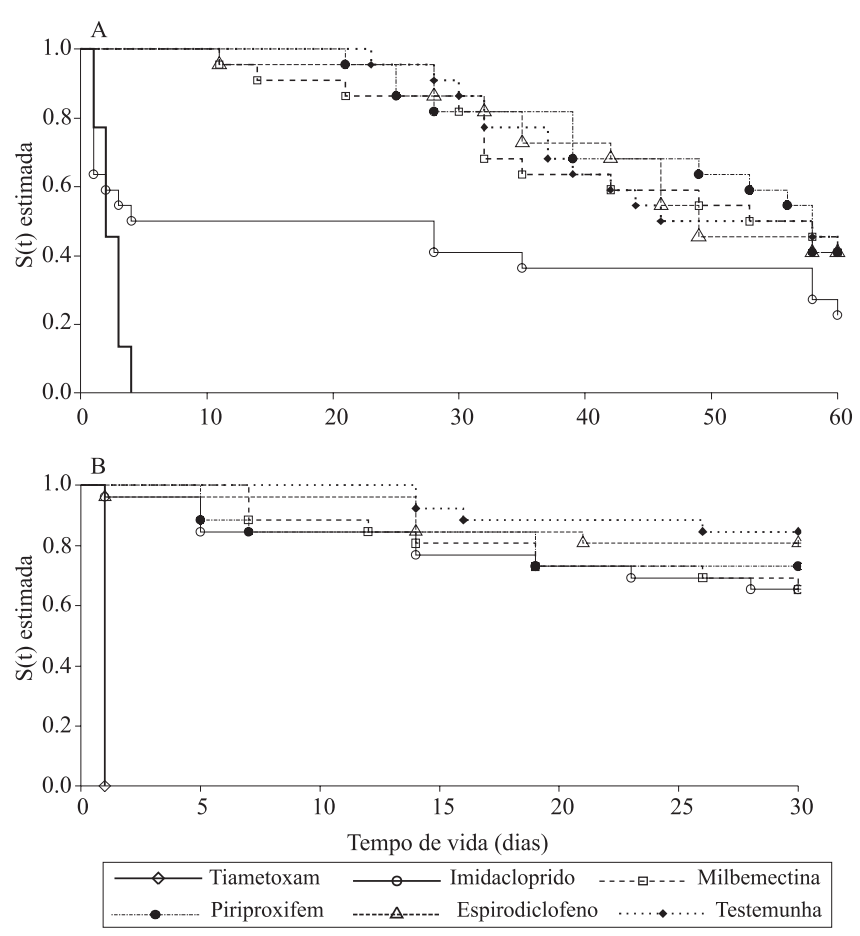

Figura 1. Funções de sobrevivência para adultos de Chrysoperla externa (A) e Ceraeochrysa cubana (B), tratados diretamente com os produtos químicos. bioensaios são realizados em condições de laboratório e com a máxima exposição dos insetos aos resíduos dos produtos, é possível constatar que os inseticidas avaliados, com exceção do tiametoxam, apresentam baixa toxicidade a essa espécie.

Quanto ao número de ovos de C. cubana, todos os compostos ocasionaram diminuição na capacidade de oviposição das fêmeas (Tabela 1). O espirodiclofeno foi o produto que mais afetou essa característica biológica. Mesmo com a diminuição de fecundidade, constatouse que todos os produtos possibilitaram médias acima de $96 \%$ para viabilidade de ovos de C. cubana; porém, apenas o imidacloprido proporcionou viabilidade significativamente semelhante à da testemunha (Tabela 1).

Santa-Cecília et al. (1997) constataram que apenas o óxido de fembutatina $\left(0,25 \mathrm{~g} \mathrm{~L}^{-1}\right.$ i.a. $)$ foi inócuo a adultos de C. cubana, tendo proporcionado $100 \%$ de sobrevivência e $99 \%$ de viabilidade dos ovos. Porém, de acordo com esses autores, os produtos fempropatrina, fenitrotiom e fenvalerato apresentaram mortalidades que variaram de 70 a $100 \%$ dos adultos e $0 \%$ de viabilidade de ovos. A semelhança de polaridades desses produtos químicos com as substâncias que compõem a cutícula dos insetos pode ser uma das causas de sua maior toxicidade.

Em virtude dos altos índices de mortalidade de ambas as espécies, ocasionados até o quarto dia após exposição dos adultos, o inseticida tiametoxam foi enquadrado na classe 4, como nocivo (Tabela 2). O imidacloprido foi categorizado na classe 3 , para $C$. externa.

Rocha (2008) relatou mortalidade de 100\% de adultos de C. externa, em razão da aplicação de imidacloprido. Essa variação na toxicidade desse produto possivelmente foi decorrente da maior dosagem utilizada por esse autor, dez vezes superior à utilizada neste estudo.

$\mathrm{O}$ tiametoxam foi o único produto que ocasionou $100 \%$ de mortalidade dos adultos de C. cubana em até 24 horas após a aplicação e, dessa forma, não foi possível avaliar os efeitos subletais desse produto. O espirodiclofeno foi categorizado na classe 2, para essa espécie, principalmente por afetar o número de ovos por dia por fêmea (Tabela 1). Os demais compostos foram classificados como inócuos aos adultos desse predador, e foram categorizados na classe 1 (Tabela 2).

Com base nos resultados de toxicidade dos compostos sobre C. externa e C. cubana, apenas 
Tabela 2. Mortalidade (M) e mortalidade corrigida $(\mathrm{Mc})^{(1)}$ provocada pelos produtos químicos quando aplicados sobre adultos de Chrysoperla externa e Ceraeochrysa cubana, número médio de ovos por dia por fêmea (R1), fertilidade dos ovos (R2), e efeito total (E) dos tratamentos sobre a fase adulta dos predadores, seguido pela classificação de toxicidade dos produtos químicos.

\begin{tabular}{|c|c|c|c|c|c|c|c|c|}
\hline Tratamento & $\begin{array}{l}\text { População inicial } \\
\text { (Adultos) }\end{array}$ & $\begin{array}{c}\text { Adultos mortos } \\
(24 \mathrm{~h})\end{array}$ & $\begin{array}{c}\mathrm{M} \\
(\%)\end{array}$ & $\begin{array}{l}\mathrm{Mc} \\
(\%) \\
\end{array}$ & R1 & $\begin{array}{l}\mathrm{R} 2 \\
(\%)\end{array}$ & $\begin{array}{c}\mathrm{E} \\
(\%) \\
\end{array}$ & Classe $^{(2)}$ \\
\hline & \multicolumn{8}{|c|}{ Chrysoperla externa } \\
\hline Tiametoxam & 22 & 5 & 22,72 & 22,72 & - & - & 100,00 & 4 \\
\hline Imidacloprido & 22 & 8 & 36,36 & 36,36 & 4,71 & 36,00 & 95,10 & 3 \\
\hline Milbemectina & 22 & 0 & 0,00 & 0,00 & 18,45 & 92,25 & 22,30 & 1 \\
\hline Piriproxifem & 22 & 0 & 0,00 & 0,00 & 28,17 & 96,08 & 0,00 & 1 \\
\hline Espirodiclofeno & 22 & 0 & 0,00 & 0,00 & 24,42 & 78,75 & 12,10 & 1 \\
\hline \multirow[t]{2}{*}{ Testemunha } & 22 & 0 & - & - & 22,11 & 99,00 & - & - \\
\hline & \multicolumn{8}{|c|}{ Ceraeochrysa cubana } \\
\hline Tiametoxam & 26 & 26 & 100,00 & 100,00 & - & - & 100,00 & 4 \\
\hline Imidacloprido & 26 & 1 & 3,85 & 3,85 & 13,44 & 98,58 & 20,80 & 1 \\
\hline Milbemectina & 26 & 0 & 0,00 & 0,00 & 12,54 & 96,25 & 25,00 & 1 \\
\hline Piriproxifem & 26 & 0 & 0,00 & 0,00 & 12,24 & 98,17 & 25,30 & 1 \\
\hline Espirodiclofeno & 26 & 1 & 3,85 & 3,85 & 9,79 & 98,42 & 42,40 & 2 \\
\hline Testemunha & 26 & 0 & - & - & 16,11 & 99,83 & - & - \\
\hline
\end{tabular}

${ }^{(1)}$ Abbott (1925). ${ }^{(2)}$ Classe de toxicidade preconizada pela IOBC (Hassan \& Degrande, 1996): classe 1, inócuo (E<30); classe 2, levemente nocivo (30 $\left.\leq \mathrm{E} \leq 79\right)$; classe 3, moderadamente nocivo $(80 \leq \mathrm{E} \leq 99)$; e classe 4, nocivo ( $\mathrm{E}>99)$.

milbemectina, piriproxifem e espiridiclofeno devem ser recomendados para uso em programas de manejo integrado de pragas, visando à manutenção e conservação dessas espécies de artrópodes benéficos em áreas de citricultura. Para os demais produtos avaliados, há necessidade de novos estudos de toxicidade em condições de semicampo e campo, haja vista que, nessas condições, geralmente existem áreas de escape para os insetos benéficos, bem como maior probabilidade de degradação dos compostos pela temperatura, luminosidade e umidade.

\section{Conclusão}

Os inseticidas milbemectina, piriproxifem e espirodiclofeno são inócuos a Chrysoperla externa e Ceraeochrysa cubana e podem ser recomendados em programas de manejo integrado de pragas visando à manutenção dessas espécies de artrópodes benéficos em áreas de citricultura.

\section{Agradecimentos}

Ao Conselho Nacional de Desenvolvimento Científico e Tecnológico e à Fundação de Amparo à Pesquisa do Estado de Minas Gerais, pelo suporte financeiro.

\section{Referências}

ABBOTT, W.S. A method of computing the effectiveness of an insecticide. Journal of Economic Entomology, v.18, p.265-267, 1925.

BEZERRA, G.C.D.; SANTA-CECÍLIA, L.V.C.; CARVALHO, C.F.; SOUZA, B. Aspectos biológicos da fase adulta de Chrysoperla externa (Hagen, 1861) (Neuroptera: Chrysopidae) oriunda de larvas alimentadas com Planococcus citri (Risso, 1813) (Hemiptera: Pseudococcidae). Ciência e Agrotecnologia, v.30, p.603-610, 2006.

BUENO, A.F.; FREITAS, S. Efeito do hexythiazox e imidacloprid sobre ovos larvas e adultos de Chrysoperla externa (Hagen) (Neuroptera: Chrysopidae). Revista Ecossistema, v.26, p.74-77, 2003.

CARVALHO, C.F.; SOUZA, B. Métodos de criação e produção de crisopídeos. In: BUENO, V.H.P. (Ed.). Controle biológico de pragas: produção massal e controle de qualidade. Lavras: Ufla, 2000. p.91-109.

CARVAlHO, G.A.; GODOY, M.S.; PEDROSO, E.C. Uso da seletividade de inseticidas e acaricidas no manejo integrado de pragas de hortaliças. In: ZAMBOLIM, L.; LOPES, C.A.; PICANÇO, M.C.; COSTA, H. (Ed.). Manejo integrado de doenças e pragas: hortaliças. Viçosa: UFV, 2007. p.539-575.

COSTA, R.I.F.; CARVALHO, C.F.; SOUZA, B.; LORETI, J. Influência da densidade de indivíduos na criação de Chrysoperla externa (Hagen, 1861) (Neuroptera: Chrysopidae). Ciência e Agrotecnologia, p.1539-1545, 2003. Edição especial.

GITIRANA NETO, J.; CARVALHO, C.F.; SOUZA, B.; SANTA-CECÍLIA, L.V.C. Flutuação populacional de espécies de Ceraeochrysa Adams, 1982 (Neuroptera: Chrysopidae) em 
citros, na região de Lavras-MG. Ciência e Agrotecnologia, v.25, p.550-559, 2001.

GODOY, M.S.; CARVALHO, G.A.; MORAES, J.C.; COSME, L.V.; GOUSSAIN, M.M.; CARVALHO, C.F.; MORAES, A.A. Seletividade de seis inseticidas utilizados em citros a pupas e adultos de Chrysoperla externa (Hagen) (Neuroptera: Chrysopidae). Neotropical Entomology, v.33, p.359-364, 2004.

HASSAN, S.A.; DEGRANDE, P.E. Methods to test the side effects of pesticides on Trichograma. In: PARRA, J.R.P.; ZUCCHI, R. (Ed.). Curso de controle biológico com Trichogramma. Piracicaba: FEALQ, 1996. p.63-74.

INTERNATIONAL ORGANIZATION FOR BIOLOGICAL CONTROL. West Palaearctic Regional Section. Working Group "Pesticides and Beneficial Organisms". Guidelines for testing the effects of pesticides on beneficial organisms: description of test methods. IOBC/WPRS Bulletin, v.15, p.1-186, 1992.

MOURA, A.P.; CARVALHO, G.A.; MOSCARDINI, V.F.; MARQUES, M.C.; SOUZA, J.R. Toxicidade de pesticidas recomendados na produção integrada de maçã (PIM) a populações de Chrysoperla externa (Hagen) (Neuroptera: Chrysopidae). Neotropical Entomology, v.38, p.395-404, 2009.

R DEVELOPMENT CORE TEAM. R: a language and environment for statistical computing. Vienna: R Foundation for Statistical Computing, 2010. Available at: <http://www.R-project.org>. Accessed on: 22 Nov. 2008.

RIBEIRO, L.J.; BERTI FILHO, E.; MACEDO, L.P.M.; MAGRO, S.R. Predação da lagarta-minadora-dos-citros Phyllocnistis citrella Stainton, 1856 (Lepidoptera: Gracillariidae) por larvas de Chrysoperla externa (Hagen 1861) (Neuroptera: Chrysopidae). Revista Caatinga, v.20, p.100-105, 2007.

RIBEIRO, M.J.; CARVALHO, C.F.; MATIOLI, J.C. Influência da alimentação larval sobre a biologia de adultos de Chrysoperla externa (Hagen, 1861) (Neuroptera: Chrysopidae). Ciência e Prática, v.15, p.349-354, 1991.
RIPPER, W.E.; GREENSLADE, R.M.; HARTLEY, G.S. Selective insecticides and biological control. Journal of Economic Entomology, v.44, p.448-459, 1951.

ROCHA, L.C.D. Seletividade fisiológica de inseticidas utilizados em cultura cafeeira sobre os predadores Chrysoperla externa (Hagen, 1861) (Neuroptera: Chrysopidae) e Cryptolaemus montrouzieri Mulsant, 1853 (Coleoptera: Coccinellidae). 2008. 133p. Tese (Doutorado)-Universidade Federal de Lavras, Lavras.

SANTA-CECÍlIA, L.V.C; SOUZA, B.; CARVALHO, C.F. Seletividade de alguns inseticidas/acaricidas aos adultos de Ceraeochrysa cubana (Hagen, 1861) (Neuroptera: Chrysopidae) em laboratório. Pesquisa Agropecuária Brasileira, v.32, p.803-806, 1997.

SAS INSTITUTE. SAS/STAT: user's guide. Cary: SAS Institute, 2001. 502p.

SCHUSTER, D.J.; STANSLY, P.A. Response of two lacewing species to Biorational and broad-spectrum insecticides. Phytoparasitica, v.28, p.297-304, 2000.

SOUZA, B.; SANTA-CECÍLIA, L.V.C.; CARVALHO, C.F. Seletividade de alguns inseticidas e acaricidas a ovos e larvas de Ceraeochrysa cubana (Hagen 1861) (Neuroptera: Chrysopidae) em laboratório. Pesquisa Agropecuária Brasileira, v.31, p.775-779, 1996.

TOMIZAWA, M.; CASIDA, J.E. Neonicotinoid insecticide toxicology: mechanisms of selective action. Annual Review Pharmacology Toxicology, v.45, p.247-248, 2005.

TUKEY, J.W. The technical tools of statistics. The American Statistician, v.19, p.23-28, 1965.

VOGT, H. Untersuchungen zu Nebenwirkungen von Insektiziden und Akariziden auf Chrysoperla carnea (Stephens) (Neuroptera: Chrysopidae). Mededelingen van de Faculteeeit van de Universite de Gent, v.57, p.559-567, 1992.

Recebido em 22 de abril de 2010 e aprovado em 22 de outubro de 2010 